\title{
Shaping, Forming and Modeling of Advanced High Strength Steel
}

\author{
MARK R. STOUDT ${ }^{1,2}$ \\ 1.--Material Measurement Laboratory, National Institute of Standards and Technology, \\ Gaithersburg, MD, USA. 2.—e-mail: stoudt@nist.gov
}

Throughout the transportation industry, there is a need to reduce vehicle weight and improve fuel economy, which has heightened the demand for new sheet alloys. The numerous advances in alloy design and production have produced numerous advanced high-strength steel (AHSS) sheet alloys that demonstrate an excellent combination of properties. In most cases, AHSS alloys demonstrate superior strength compared to the traditional alloys used for automobile components, and for this reason, replacing components made from traditional steel alloys with those made from AHSS is a good strategy to achieve weight reduction. However, the property data and constitutive laws available for many of these alloys are limited or inadequate for use in the complex models used to predict the behavior during sheet metal forming. The ensuing inability to accurately predict the deformation behavior during forming impedes widespread incorporation of these advanced alloys in many applications.

The enhanced mechanical properties associated with AHSS alloys are largely achieved through complex phase transformations and heat-treatment protocols. Since the microstructure determines the properties, a fundamental understanding of the microstructural evolution in AHSS alloys is an essential component for further refining the properties of existing alloys, or for developing new alloys. The papers in this section highlight some recent advances pertaining to the characterization, forming, and modeling of AHSS alloys.

The first paper, "Measuring the Influence of Pearlite Dissolution on the Transient Dynamic Strength of Rapidly-Heated Plain Carbon Steels," by Mates et al. describes a series of high strain rate compression measurements that were conducted while the steel was undergoing transformation from

Mark R. Stoudt is a past-Chair of the Shaping and Forming Committee of the TMS Materials Processing \& Manufacturing Division, and guest editor for the topic Shaping, Forming, and Modeling of Advanced High-Strength Steels in this issue. a lamellar pearlite/ $\alpha$-ferrite structure to austenite. The authors conducted measurements in a model system that consisted of three plain carbon steels with differing carbon contents. Their results demonstrated that the kinetics of the pearlite to austenite transformation are extremely fast, and that the strength of the steel is substantially reduced during the transformation due to the transient strength effect produced by the decomposition of the pearlite.

The second paper, "Ductility of Advanced High Strength Steel in the Presence of a Sheared Edge," by Ruggles et al. describes how the ductility of the steel sheet is influenced in the vicinity of a sheared edge. The authors conducted experiments on two AHSS alloys with similar strengths, i.e., Dual Phase 980 (DP980) and Transformation-Induced Plasticity-Assisted Bainitic-Ferritic 980 (TBF980), using both a standard tensile test protocol and an incremental tensile straining technique developed for use in a scanning electron microscope. Digital image correlation was used to determine the changes in the effective strain at the microstructural level for both measurements. Their results revealed that TBF980 had significantly better ductility than DP980 due to a higher propensity for localization around the hard phases present in the DP980. This effect was exacerbated in the vicinity of a sheared edge.

The third paper, "Evaluation of Springback for DP980 S Rail using Anisotropic Hardening Models," by Choi et al., reports the results of their modeling of the springback (i.e., the unexpected change in shape that occurs in a component after forming) in an automotive "S-rail" component. The authors compared the deformation behavior predicted by two constitutive models, the Yoshida-Uemori model and the homogeneous anisotropic hardening (HAH) model, against the physical measurements taken from an S-rail stamped from a DP980 steel sheet. Both models are based on isotropic and kinematic hardening and are designed to capture the Bauschinger effect, transient hardening and 
permanent softening that occurs after there is a change in the strain path. Their results demonstrated that, of the springback prediction models evaluated, the HAH model best agreed with the experimental results.

The following papers being published under the topic of Shaping, Forming and Modeling of Advanced High Strength Steel provide recent advancements on this subject. To download any of the papers, follow the url: http://link.springer.com/ journal/11837/68/07/page/ 1 to the table of contents page for the July 2016 issue (vol. 68, no. 07).
- "Measuring the Influence of Pearlite Dissolution on the Transient Dynamic Strength of RapidlyHeated Plain Carbon Steels," Steven Mates, Mark Stoudt, and Sindhura Gangireddy.

- "Ductility of Advanced High-Strength Steel in the Presence of a Sheared Edge," Tim Ruggles, Stephen Cluff, Michael Miles, David Fullwood, Craig Daniels, Alex Avila, and Ming Chen.

- "Evaluation of Springback for DP980 S Rail Using Anisotropic Hardening Models," Jisik Choi, Jinwoo Lee, Gihyun Bae, Frederic Barlat, and Myoung-Gyu Lee. 